\title{
EL FANTASMA DE LAS GRIETAS: UNA ESPECULACIÓN A-HISTÓRICA
}

\author{
Enrique V. Nuesch ${ }^{*}$ \\ Universidade Estadual do Paraná \\ Universidade Estadual de Londrina
}

\begin{abstract}
Resumen: Partiendo del concepto de "forma del tiempo", de Siegfried Kracauer y George Kubler, ensayamos una lectura de la recepción de la imagen mutilada del cuerpo humano en el arte, analisando cómo en torno a esa imagen se articulan discursos de historiadores, artistas y críticos en ciertos momentos de la historia. Sugerimos que junto con Agamben, Derrida y Deleuze se puede pensar en un fantasma que acecha a la imagen del cuerpo en el arte, cuya espectralidad se manifiesta bajo los discursos que tratan de reprimirla. Visitamos el Renacimiento, el Siglo XVIII y el pasaje del XIX al XX. Se concluye que una mudanza de actitud en el XX es reflejo de la reversión del platonismo que se anunciaba desde el XIX, pero cuyos síntomas se hacían sentir desde antes, manifestados de forma contradictoria en discursos que elogian la belleza del fragmento al mismo tiempo en que de él se sirven para alcanzar una imagen de lo bello más allá de lo material.

Palabras clave: Arte. Cuerpo. Imagen. Fantasma. Historia.
\end{abstract}

\section{Introducción}

Borges, con su famoso Funes, nos ha propuesto la experiencia de cómo sería una vida sin olvidar siquiera un detalle, un fragmento del tiempo, un hecho, un perfil de lo que se nos muestra en el mundo, una letra de lo que se nos presenta en un libro. En este último aspecto, la memoria de Funes puede tener algo de la babélica biblioteca también ideada por Borges: todo lo que en ella entra, ahí se queda, todo lo que registran sus libros, igualmente, y todo lo que puedan prever los mismos, por sueño, profecía o hipótesis científica, también, todo ahí se queda. Si viva fuese la biblioteca, funesiana sería, y no del todo absurdo es

\section{Esta obra está licenciada sob uma Creative Commons - Atribuição 4.0}

\footnotetext{
* Professor Assistente na UNESPAR (Universidade Estadual do Paraná), Campus de Apucarana, PR. Doutorando em Estudos Literários pela UEL (Universidade Estadual de Londrina). Possui graduação em Letras Língua Espanhola e Literaturas pela Universidade Federal de Santa Catarina (2004) e Mestrado em Teoria Literária pela mesma instituição (2007). Foi Professor Assistente I do curso de Letras: Língua e Literatura Portuguesa e Língua e Literatura Espanhola, Universidade Federal do Amazonas (Instituto de Natureza e Cultura de Benjamin Constant) entre 2009 e 2011. Tem experiência na área de Letras com ênfase em Teoria do Texto Digital e no ensino de literaturas de língua espanhola e teoria literária. E-mail: enriquenuesch@gmail.com
} 
suponerla viva, por su constante movimiento, deslizando sus registros (y dentro de los registros, los registros que cifran los registros), como se dislocan en una memoria los vividos y no vividos, por represión, nachträglichkeit o différance.

¿Qué contagio se puede hacer de esta imagen borgeana con la Historia? Talvez siguiendo a Jaime Concha, en su conocido "Borges y la Historia”, que nos muestra cómo en la "Postdata" al último cuento de El Aleph, las palabras del narrador sugieren la existencia de una "reversibilidad histórica, con la instalación de un tiempo regresivo en la historia de la humanidad" (CONCHA, 1983, p. 484); así, la Biblioteca y Funes, en sus omniregesta, nos mostrarían, la primera, que todo registro, en sus infinitas combinatorias, excede el tiempo registrado, el segundo, que el recuerdo de un día excede un día, pues hay que recordar lo que fue recordado a lo largo del día que se trata de recordar: la Historia arrastra en su recorrido supuestamente lineal un halo de la discontinuidad de sus excesos anacrónicos, cuyas señas abren el camino hacia el tiempo regresivo, que transporta del periodo historiado hacia un nohistoriado, que rehuye a cualquier intento de una aufhebung narrativa e insiste en su presencia en un tiempo que no le corresponde. Por ello, quizá, a este tiempo se haga referencia en la forma de un otro, reprimido bajo la tradición, y alcanzado forzosamente, por deconstrucción o violencia hermenéutica, como lo hacen Derrida con la nachträglichkeit freudiana y Deleuze con el aiôn estoico, o por alegoría, como lo hace Siegfried Kracauer, al invocar a la figura del Judío Errante, Ahasuerus. Éste, Kracauer, con su remisión a Ahasuerus, será nuestro guía en la apertura del camino que proponemos en este breve ensayo. Trataremos de considerar el tema que el presente número de Anuário de Literatura propone bajo la perspectiva de una especie de esta "temporalidad regresiva", en la que posibles fantasmas de la imagen del cuerpo pueden asomarse $\mathrm{y}$, ocasionalmente, imponerse, en franca pugna con la imagen idealizada de lo bello a lo largo de la historia. Para construir nuestras razones, haremos algunos "saltos temporales" por la historia del arte occidental y los discursos que la animan, así como nos apoyaremos sobre algunas estrategias de lectura delineadas por pensadores como Agamben, Deleuze y Derrida.

\section{El Ahasuerus de Kracauer}

Siegfried Kracauer falleció al fin de 1966 y dejó, para sumarse a su ya vasta y conocida bibliografía acerca de la imagen y del cine, las anotaciones y capítulos para un libro que poco tiempo después vio la imprenta, History: The last things before the last (1969). Unos pocos puntos anecdóticos pueden recordarnos algo de su posición como pensador en el siglo XX, su proximidad y, al mismo tiempo, lejanía de su discípulo Th. Adorno, con quien 
(cuenta el frankfurtiano) leía en matinales sábados la primera Crítica kantiana (ADORNO, 1974, p. 388) y compartía un antihegelianismo (y en esto se incluye a Benjamin, amigo de Kracauer) (RODOWICK, 1987, p. 120), pero, por otro lado, del que disentía totalmente en la concepción de una dialéctica que a todo penetrara, sin mínimo espacio para una ontología (KRACAUER, 1969, p. 201). Esta disensión en particular se hace por sobre el trasfondo de una idea del tiempo que Kracauer trata de establecer en el capítulo cuarto de su libro póstumo. Cuando critica a la dialéctica adorniana, lo hace en el proceso de una búsqueda por lo que pudiese ser la faceta histórica de las verdades filosóficas, colocando y desechando en seguida la alternativa entre trascendentalismo e imanentismo, el segundo representado por Adorno y el primero por Loewith. En la perspectiva de Kracauer, ambos se inclinan o a una ontología absoluta (el primero) o a un flujo histórico sin dirección ni espacio para cualquier juicio determinado (el segundo). Al desechar la alternativa, lo que propone es que se consideren ambas posiciones, en una fórmula de que el mismo autor admite la dificultad de imaginar; de todos modos, así queda la proposición:

From the angle of my proposition, philosophical truths have a double aspect. Neither can the timeless be stripped of the vestiges of temporality, nor does the temporal wholly engulf the timeless. Rather, we are forced to assume that the two aspects of truths exist side by side, relating to each other in ways which I believe to be theoretically undefinable (KRACAUER, 1969, p. 200).

El intento de seguir en la labor de darle formulación más precisa a este modo de tratar las verdades filosóficas no nos interesa para lo que sigue, sino, antes, la propia concepción del tiempo que da base al planteamiento. Si las verdades filosóficas deben tener un "doble aspecto", es porque tal duplicidad les es imprimida por el mismo tiempo en el que necesariamente se construyen. Ahí es donde nos encontramos con la figura de Ahasuerus.

La figura del "Judio Errante" surge para darle nombre al capítulo en que Kracauer se dispone a construir su concepción del tiempo histórico, "Ahasuerus, or the Riddle of Time". El Ahasuerus de Kracauer no se reporta a las historias del Antiguo Testamento, en las que se nombra a un Ahasuerus relacionado a la realeza hebrea, ni siquiera a otra fuente explícitamente mencionada; el hecho de que el autor mencione la "leyenda" de Ahasuerus indica con bastante seguridad que se trata de una leyenda cristiana popularizada en el siglo XIII, directamente relacionada con el castigo de Caín (errar por la tierra por asesinar a Abel), sobre un judío que habría insultado a Jesús cuando éste cargaba el instrumento de su tortura y postrero símbolo; por ello, tendrá que vagar por la tierra hasta la segunda venida del Cristo (BAILLIE, 2014, p. 83). En la imagen que se hace Kracauer, un hombre como este está condenado a presenciar todos los acaeceres de la historia, recoger en su faz las marcas de los 
diferentes periodos que vivió y tratar de encontrar el gran flujo temporal único que constituye su vida errante:

\begin{abstract}
He indeed would know firsthand about the developments and transitions, for he alone in all history has had the unsought opportunity to experience the process of becoming and decaying itself. (How unspeakably terrible he must look! To be sure, his face cannot have suffered from aging, but I imagine it to be many faces, each reflecting one of the periods which he traversed and all of them combining into ever new patterns, as he restlessly, and vainly, tries on his wanderings to reconstruct out of the times that shaped him the one time he is doomed to incarnate) (KRACAUER, 1969, p. 157).
\end{abstract}

Ahasuerus, pues, encarna la misma "duplicidad" del tiempo a que arriba se hacía referencia. Kracauer lo toma como una salida para expresar su idea conflictiva de cómo se debe entender y registrar la temporalidad histórica, que no acepta alternativas, sino la misma conjunción entre dos formas de registro, que puedan comprender lo cronológico y lo anacrónico:

Thus we are confronted with two mutually exclusive propositions neither of which can be dismissed. On the one hand, measurable time dissolves into thin air, superseded by the bundles of shaped times in which the manifold comprehensible series of events evolve. On the other, dating retains its significance inasmuch as these bundles tend to coalesce at certain moments which then are valid for all of them (KRACAUER, 1969, p. 154).

Por un lado, los agrupamientos de "tiempos formados" (shaped times) impiden que una temporalidad cronológica se afirme, en la medida en que un periodo no puede ser explicado solamente por su contexto, sino que necesariamente envía a otro, que no le es inmediatamente anterior ni posterior, sin cualquier línea de continuidad; por otro lado, la cronología puede ser considerada en la medida en que tales agrupamientos por veces convergen de forma en que se exibe una unidad para el periodo. Evidentemente, esto no cobra sentido si no explicamos qué quiere decir la expresión "tiempos formados". Es una que Kracauer retoma de su maestro George Kubler, el cual la acuñó en su estudio The Shape of Time ([1962] 2008). Con su mirada hacia la historia del arte, Kubler propuso que las cosas (el subtítulo del libro es "remarks on the history of things") poseen en su constitución temporalidades distintas, que corresponden a diversos periodos de la historia y, por lo tanto, la noción de "periodo" necesita ser repensada:

Thus every thing is a complex having not only traits, each with a different systematic age, but having also clusters of traits, or aspects, each with its own age [...] historic time seems to be composed of many envelopes, in addition to being mere flow from future to past through the present (KUBLER, [1962] 2008, p. 9091). 
Así, por ejemplo, el problema de la iluminación de paisajes en pintura constituye una "forma del tiempo" que no corresponde con la historia de la pintura como usualmente se la periodiza; en su ritmo e intervalos, el problema forma su propio tiempo, surgiendo, desapareciendo y resurgiendo en momentos cronológicamente distintos

The anonymous mural painters of Herculaneum and Boscoreale connect with those of the seventeenth century and with Cézanne as successive stages separated by irregular intervals in a millenary study of the luminous structure of landscape, which probably will continue for many generations more upon equally unpredictable rhythms (KUBLER, [1962] 2008, p. 80).

En esta perspectiva, los paisajes de Cézanne participan en una otra "forma del tiempo", además del propio tiempo cronológico que se les adjudica en la historia del arte. Por ello, como quiere Kracauer, no hay pasaje continuo entre periodos e, incluso, algunos pueden surgir de parte alguna. Un recorrido histórico, pues, bien puede hacerse a los saltos, por encima de la línea evolutiva y temporal de una historia continua:

\footnotetext{
You must, so to speak, jump from one period to another. That is, the transitions between successive periods are problematic. There may be breaks in the process; indeed, a period may, as an emergent 'event' in Focillon's sense, arise from 'nowhere' (KRACAUER, 1969, p. 155).
}

Si el tiempo histórico no se permite mirar sino como las cambiantes faces de Ahasuerus, la mirada sobre las cosas de la historia que en su rostro se exhiben debe ver objetos calidoscópicos, no en la figura plana del juguete que en las infancias se hacen con abigarradas piedras, sino en una profundidad, que nos remite de forma a forma, de un entonces a otro, en una deriva sin término posible que no el que le dé uno mismo. A una de estas derivas nos interesa aventarnos ahora, tratando de vislumbrar la imagen fugaz aparente bajo la superficie de unas pocas esculturas, un fantasma que hace su eco en el calidoscopio temporal, que creemos poder capturar vagamente, si no por el intento de nuestra propia mirada, en los discursos que plasmaron su aparecer.

\section{La rota blancura del mármol}

Si registran con exactitud los cronistas del Renacimiento, de antaño y contemporáneos, el aparecimiento de las esculturas fragmentarias de la Antigüedad en las primeras décadas del siglo XV fue mucho más que un hecho arqueológico y su influencia en el mundo artístico de entonces es más profunda que la vulgata del "entusiasmo" por el estudio de la antigüedad grecolatina. Del Torso Belvedere, sobre el que aun se diputa si es del periodo romano o helenístico, se dice que el registro más temprano encontrado de su aparecimiento se 
dio por 1430 (CHILVERS, 2007, p. 55). Sin lugar a discusiones, sin embargo, es que ante la figura de esta escultura rota se convino que no sería restaurada, o por lo menos no se lo intentó en su momento de aparición, ni en seguida; lo contrario, por ejemplo, que sucedió a otra escultura fragmentaria hallada en el mismo momento, el Lacoön. Y dice la crónica que el Torso impactó profundamente a uno de los grandes maestros, Michelangelo Buonarroti: la visión del fragmento se manifiesta en algunas de sus obras, como el ignudo en uno de los afrescos del techo de la Capilla Sistina (por encima y a la derecha de Jeremías), en la escultura Día en la tumba de los Medici y en Jesús y San Bartolomeo en la pintura Juicio Final; el mismo Lacoön fue influencia para otra serie de obras (SCHARFSTEIN, 2009, p. 169). Lo fragmentario, pues, fue un propulsor para el maestro florentino en una parte importante de su labor artística, lo que ha delineado una idea de la misma imaginación artística que lo habría guiado. Por ejemplo, en la crónica contemporánea del Renacimiento, Leonard Barkan lo describe como el "héroe trágico del fragmento":

If the Torso Belvedere is the paradigm of ancient sculpture and Michelangelo is the tragic hero of the fragment, that is because he based his career in part on what could be imagined and not seen or because he appeared too often to be unable to produce statues more complete than the fragments that inspired him (BARKAN, 1999, p. 207)

Una descripción que nos sugiere que, del material deteriorado que le exhibe un cuerpo incompleto, el maestro completaba a través de su imaginación el bello cuerpo que terminaba en los bordes rotos. Aún ante un bloque de mármol nuevo, que no ha todavía recibido los golpes que le arrancarían una escultura, dice otro cronista de nuestro tiempo como Ernst Gombrich, que Buonarroti abstraía la forma en potencia que moraría en la roca, en la espera por su emergencia: "[Michelangelo] always tried to conceive his figures as lying hidden in the block of marble on which he was working; the task he set himself as a sculptor was merely to remove the stone which covered them" (GOMBRICH, 1989, p. 313). Completando la figura con la mirada, o imaginando la figura dentro de un bloque, del cual nomás se quitan los excesos que se depositan sobre la figura, se sabe que el origen de esta idea le viene de una concepción neoplatónica del mundo, según la cual el alma se encuentra encerrada en la materia, que le es ontológicamente inferior, y, por lo tanto, la bella forma se encuentra encerrada en la piedra, expectante por su "liberación" a manos del escultor. A Michelangelo le llega por el contacto con el pensamiento de Marsilio Ficino, el neoplatónico que frecuentaba a los Medici (JANSON, 2004, p. 437). Como han señalado cronistas del maestro (TAYLOR, 1840, p. 78; STOKES, [1955] 2002, p. 77), los versos de un famoso soneto suyo declaran su forma de ver la cuestión, cuando escribe "Non ha l'ottimo artista 
alcun concetto, / Ch'un marmo solo in sè non circonscriva" (BUONARROTI, [15--?] 1904, p. 17). Asimismo, en una carta a Benedetto Varchi, afirma que la escultura es el arte de "retirar": "Io intendo scultura, quella che si fa per forza di levare" (BUONARROTI [1549] 1875, p. 522). Aun siendo conocida esta perspectiva suya, otros testimonios de la propia época del artista, indican que no era sólo de él, sino más como un producto del Zeitgeist del momento. Unos versos coligidos por Giorgio Vasari, dedicados por el poeta Giambattista Strozi a una copia de la Pietá instalada en la iglesia del Santo Spirito en 1549, transpiran la idea de que bajo el muerto mármol late la vida por la forma que participa de la idea de lo bello:

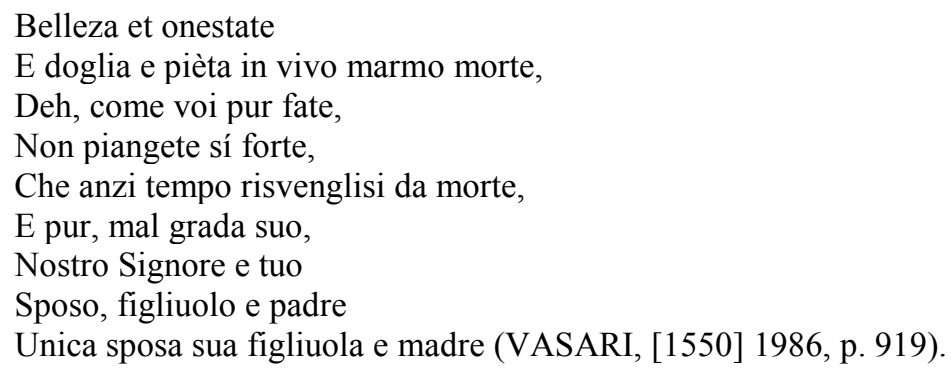

$\mathrm{Si}$, por un lado estas manifestaciones discursivas individuales de ese momento se armonizan con las de nuestro tiempo acerca del mismo, por otro, esto no nos muestra lo que podría haber de conflictivo en la mirada sobre los mármoles rotos. Dijimos que el hallarse de las antigüedades fue más allá que la simple inspiración para el humanismo y la actitud que aspiraba a lo clásico grecolatino. El Torso Belvedere ya había sido, antes de que se volviese el "torso de Michelangelo", conocido por, entre otros, un gran artista como Leon Battista Alberti, setenta años mayor que Michelangelo. Y sobre Alberti, el fragmento no tuvo el impacto que se vio en Michelangelo, por lo contrario, le pareció casi antinatural la torsión corporal sugerida por el torso y nada al gusto de su época (SUMMERS, 1977, p. 339). Por ello, tal vez sea interesante pensar que subterráneamente al impacto del Torso como fuente de inspiración y ascensión hacia la idea de lo bello - o sea, de su capacidad de conducir la mirada de lo material hacia la visión de lo ideal- se esté introduciendo otra idea completamente opuesta: que ese cuerpo que se exhibe como una espantosa mutilación sea, de hecho, bello. A ésta es que Leonard Barkan se refiere como un "giro categorial" (BARKAN, 1999, p. 122), que replantearía el quehacer artístico ante la imagen de los cuerpos quebrados. Tobin Siebers (2008), a su vez, sitúa como un punto de partida del Renacimiento hacia la edad contemporánea de lo que sería la entrada de la imagen de cuerpos mutilados como lo bello artístico. A partir de la adopción del Torso Belvedere por Michelangelo, dice Siebers, se pueden detectar las señas de ese proceso subterráneo, que se empezaría de hecho a imponer en 
la superficie de lo visible solamente pasada la mitad del siglo XX como una "estética de lo inválido" (inválido como "disability", un concepto que abarca toda característica del cuerpo humano que no está en conformidad con la idea de la "normalidad" o lo "sano"). A estas señas nombra el autor por "signposts", las que, aunque él mismo no lo diga, se muestran, en su calidad de hechos de historia del arte, como los trazos anacrónicos a los que nos referíamos arriba con Kubler y Kracauer:

\footnotetext{
Signposts are often crossing points where historical forces mingle. They are at once deeply chronological and anachronistic, simultaneously historical and non-historical. They make evident that disability as a concept bears weight backwards in time, giving meaning retroactively to images and ideas for the advancement of disability aesthetics (SIEBERS, 2008, p. 331).
}

Si de hecho, como afirma este autor, la imagen del Torso y otras antigüedades rotas descubiertas cerca de la época de Michelangelo llegó a estimular incluso a que otros artistas mutilaran sus propias esculturas para tratar de imitar la belleza de lo que veían (SIEBERS, 2008, p. 330), entonces, más que un intento de alcanzar lo bello más allá de lo material, se estaba introduciendo en el dominio de lo bello la misma imagen que se trata de negar por la trascendencia de lo material a lo ideal; sin embargo, no como un acto consciente e intencional, sino como un exceso, o un fantasma, que se cuela junto con la Idea en el retorno del reino de las puras formas hacia el intelecto que lo trata de contemplar. Se cuela como el exceso anacrónico que resurge aquí y allá en la historia del arte, y se puede vislumbrar en el mismo discurso de los que tratan de realizar la mirada contemplativa.

\section{El fantasma de las grietas}

Como el mismo Siebers señala, talvez uno de los discursos más elocuentes acerca del Torso fue el de Johann Joachin Winckelmann, en su Geschichte der Kunst des Alterthum, de 1764. Pero su mención al "padre" dela historia del arte se hace en un sentido, según lo vemos, que subraya nada más que la parte positiva, es decir, el hecho de que Winckelmann se detiene ante la escultura y afirma su belleza (SIEBERS, 2008, p.331). De nuestra parte, creemos que hay algo más que leer en sus palabras. En su estudio Flesh and Ideal: Winckelmann and the origins of Art History (1994), Alex Potts hace una lectura deconstructiva de los escritos de Winckelmann, sobretodo con base en Paul de Man. Uno de sus principales logros, según nos parece, es mostrar el instante histórico que subyace bajo el discurso de Winckelmann, que lo pone en el punto de un giro conceptual que marca el pasaje de un ímpetu por afirmar los valores estáticos y eternos de la antigüedad griega a la concepción orgánica y evolutiva del arte de un romanticismo que se origina de la Ilustración. El historiador se encuentra al borde 
de ese momento y todavía trasparece una visión, por lo menos conceptualmente, no muy lejana de la que vimos en los discursos que arriba mencionamos. Una mirada a los discursos de Winckelmann muestra que ante las antigüedades griegas éste pugna por darles vida, tratando de alcanzar todo lo que podría estar contenido en la figura que se le muestra, en un esfuerzo por recolocarlas dentro de marcos o matrices mitológicos. El Torso Belvedere, para él, es el cuerpo de Héracles, y através de la piedra se puede ver al héroe y dios en acción: "Alsdann wird dir Herkules wie mitten in allen seinen Unternehmungen erscheinen, und der Held und der Gott werden in diesem Stücke zugleich sichtbar werden" (WINCKELMANN, [1764] 1870, p. 351) $)^{1}$. Aún más, con su mirada, es capaz de hacer la restauración ideal de la rota piedra, trasponiendo la grieta invisible entre lo ideal del mito y lo material del mármol dañado:

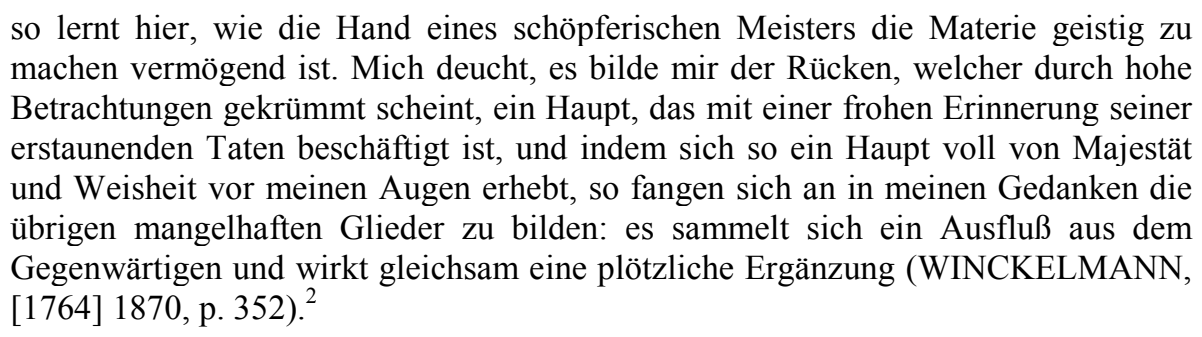

Para que la visión pueda vislumbrar el "eflujo" de la forma perfecta de la escultura es necesario que de alguna forma se haga trasparente o se anule la materia que la contiene o la encarna y exhibe con deficiencia; por ello, como señala Potts, Winckelmann tiene que dirigir su mirada desde la perspectiva de la representación, como quien tiene que hacer el salto del significante material al significado inmaterial, siguiendo el camino de la más conocida metafísica de la presencia:

like the most elevated antique ideal, functions as that necessarily problematic category of representation that would directly body forth a high idea, but in so doing would have to divest itself of its material substance so as to become transparent to its immaterial meaning (POTTS, 1994, p. 98).

Sin embargo, como en todo salto de esta naturaleza, Winckelmann tendrá que vérselas con el espectro del otro que resiste, como la contaminación de lo inmaterial por lo material. En una de las variantes de su texto acerca del Torso, entre los manuscritos hallados

\footnotetext{
1 “Entonces le aparecerá a ud. Héracles en medio a sus trabajos, y el héroe y el dios será visible de golpe en esta piedra"

2 “que se aprenda aqui como la mano de un creativo maestro es capaz de hacer espiritual a la materia. Me parece que de las espaldas, que se muestran curvadas en elevadas consideraciones, se construye una cabeza ocupada en gaudios recuerdos de sus admirables hechos; y mientras que una tal cabeza colmada de majestad y sabiduría ante mis ojos se yergue, así se construyen en mi pensamiento los demás miembros faltantes: un eflujo de lo que tengo ante mi se reúne y como que de súbito produce una restauración"
} 
en Florencia, se lee su ambigua actitud acerca del cuerpo fragmentario que tiene ante los ojos: "Wenn ich den Torso von Belvedere besehe, so weiss ich nicht, ob ich mehr traurig über den Verlust der schönen Glieder oder fröhlich über den wunderschönen Körper, so uns übrig bleibt sein soll” (WINCKELMANN, [176-] 2002, p.381)². Este “wunderschönen Körper” que al mismo tiempo lo entristece y alegra, a lo que parece, de hecho necesita ausentarse para dar lugar a su verdadera forma, que no es de este mundo material, como nos lo sugiere Potts al afirmar que para Winckelmann "the Greek ideal embodied an integrity and wholeness that in modern culture could only be experienced as absence" (POTTS, 1994, p. 47); talvez podamos decir, con un eco de Agamben, que Winckelmann repite la escena edípica del enigma, y ante la escultura, "lo que hay de inquietante y de tremendo en el enigma desaparece inmediatamente si se vuelve a llevar su decir a la transparencia de la relación entre el significado y su forma, del que sólo en apariencia éste logra escapar" (AGAMBEN, 2006, p. 232).

Un salto cronológico, o un parpadear anacrónico, hacia el fin del XIX y primeras décadas del XX nos muestra que la situación no es diferente. En el intervalo entre la época de Winckelmann y la que miramos ahora, de hecho, hubo otro acontecimiento, tal vez con la misma fuerza que el del Torso Belvedere: el desenterrar de la Venus de Milo, en 1810. Se sentirá su eco en obras de grandes maestros, como Rodin, Magritte y Maillol, en décadas posteriores. Si seguimos lar razones de Siebers, podemos decir, en contra de lo que recién afirmamos, que sí hay diferencia, que ahora, sí, la imagen de la mutilación se ha vuelto, por fin, una figura central de lo bello artístico. $\mathrm{O}$, si se quiere ser más riguroso en lo que dice respecto a las transformaciones de la estética, podemos desechar el uso de "bello", hacer hacia un lado su eco platónico y kantiano, y decir, que por lo menos, ahora la imagen de la mutilación se propone como fuente central de afectos en obras de grandes maestros. Y, sin embargo, el vislumbre del fantasma todavía acecha las sensibilidades, y se asoma entre los discursos acerca de las obras.

La crónica de Gombrich acerca de la recepción de esculturas de Rodin da una idea de cómo se veía su concepción artística en su momento. Al público general, la visión de sus esculturas “incompletas", en gran medida, no parece haber causado admiración:

Like the Impressionists, Rodin despised the outward appearance of 'finish'. Like them, he preferred to leave something to the imagination of the beholder. Sometimes he even left part of the stone standing to give the impression that his figure was just

\footnotetext{
3 “Cuando miro al Torso de Belvedere, no sé si más me entristezco con la pérdida de los bellos miembros, o si me alegro en el bellísimo cuerpo que se nos ha dejado”.
} 
emerging and taking shape. To the average public this seemed to be an irritating eccentricity if not sheer laziness" (GOMBRICH, 1989, p. 528)

En esta descripción, hecha de una perspectiva histórica, se detecta por lo menos un trazo de lo que veíamos arriba, con Michelángelo y Wickelmann: por la ausencia, de lo material incompleto, se presentifica la completud, en un acto de intelección. De hecho, en un libro publicado al año siguiente de la muerte de Rodin, Louis Weinberg hace un sumario de las razones - por decir lo mínimo, contradictorias - según las que público y conoisseurs alimentaban su antipatía por las esculturas del artista:

At one time called a cheat because of his virtuoso cleverness in representing the form, he is later called an incompetent who hides his inability under a vague dressing gown. At another time called an iconoclastic breaker of traditions, he is then dubbed a mere imitator of Angelo in the exaggeration of his modelling, or of the Greeks in his deliberately 'dismembered bodies' (WEINBERG, 1918, p. 18-19).

Lo que se nos llama la atención en esta suma, si damos crédito a Weinberg, es la última acusación, de “imitador" de los griegos en sus cuerpos deliberadamente desmembrados, en conexión con la penúltima, de "imitador" de Michelangelo por la "exageración" de su modelaje. Si recordamos que, antes del florentino, Alberti le dio las espaldas al Torso por su postura "antinatural" y en seguida justamente ésta se volvió uno de los procedimientos que eternizaron la obra del primero, que en mucho la tomó de aquel fragmento de la antigüedad, las acusación, nos parece, se sintetiza en esto: lo antinatural y desmembrado propuesto por Rodin no presentaba a sus acusadores la deseada elevación de lo material a lo ideal. Un síntoma de ello es que, para el mismo Weinberg, cuyo libro es, al fin y al cabo, encomiástico - y no deja presentar el otro lado de la recepción de Rodin, los que lo celebraron y comprendieron -, una de las más famosas esculturas del artista, L'homme qui marche, es nombrada, en la página que la ilustra, nada más que "nude study", sin el nombre que da título al estudio (WEINBERG, 1918, p.45). Tal vez, por la misma razón que Judith Cladel ([191-?] 1917), su biógrafa y cercana contemporánea, se refiere a dicha obra como a una parte de un proceso en el que se alcanzará, por fin, la forma viva de Saint Jean Baptiste:

In "The Age of Bronze" Rodin has adopted the balanced rhythm of Greek sculpture; in his new figure he passed to the Gothic equipoise, with a harmony that is less gracious, but with a reality stronger and more living. What he did not abandon was the rigorous construction and the strength of modeling which his study of the antique had given him. "The Man Walking," as well as the greater part of his subsequent works, thus exhibits the union of the two great principles of sculpture that have governed the Occidental genius.

Rodin, strengthened by study, now made a complete statue with head and arms. While he was working he discovered that his model was half a savage, still close to the animal and its ferocious instincts. Sometimes his eyes burned, his jaws, armed with powerful teeth, were thrust forward as if to bite something; he resembled a 
wolf. At other times a kind of strange passion inflamed him. His face radiated faith and will; he spoke with such energy that he seemed to be haranguing a crowd; one would have thought him a prophet of the primitive ages, a visionary bursting forth from the desert to preach and to convert the people. Rodin regarded him in amazement. It was no longer his model, but a man from the Bible; surely it was a prophet that stood before him: it was Saint John the Baptist brought back to life. The sculptor bowed before the command of nature: his statue should bear the name of the forerunner (CLADEL, [191-?] 1917).

Como persona cercana y familiar al maestro, conocía profundamente su modo fragmentario tde rabajar y sabía admirarlo. Son diversas las instancias en que las obras que tanto molestaban a los contemporáneos son elogiadas y explicadas por sus propiedades sensuales. Lo que es notable, sin embargo, es que se plasme sutilmente en su discurso un contraste entre un antes y un "ahora", "fortalecido por el estudio", que permitió el emerger de una estatua "completa, con cabeza y brazos". Si especulábamos antes entorno a una misma razón subyacente al discurso de Weinberg y Cladel, es porque ambos hablan desde una posición que comprende y admira las obras de Rodin, pero se contradice, casi imperceptiblemente, aquí por no conceder el nombre a una de sus más famosas obras (aún diez años después de su "bautismo" como obra, en 1904), allá por contrastar dos momentos de su producción dándole énfasis positivo a uno de ellos. Nos parece que aquí se muestra una resonancia de fondo, entre la animadversión de la época, relatada por Gombrich (de parte del "general public") y Weinberg, y estos ínfimos deslices que se notan en el mismo Weinberg y en Cladel. Si, como quiere Siebers, "we have to wait until Auguste Rodin to find a sculptor who revels in broken beauty, sometimes radically fragmentary, as in L'Homme qui marche, a heroic male body missing its head and arms but not its legs" (SIEBERS, 2008, p. 31), entonces el maestro podría estar en el filo de un momento en que un cambio se está dando, y la subterránea contradicción en tela es epifenómeno de dicho cambio. Así, los discursos que hacen el elogio del cuerpo fragmentario y a un mismo tiempo su sutil derogación se construirían en una especie de "contradicción coherente", como síntoma de la pérdida de un centro; y, como dice Derrida, "comme toujours, la cohérence dans la contradiction exprime la force d'un désir" (DERRIDA, 1967, p. 410). El elogio del cuerpo fragmentado, pues, se hace aún bajo la lógica de la representación, de la intelección de un eidos al que la incompletud amenaza en su plenitud ideal; mientras que el fragmento es mera etapa hacia un télos, no podrá ser aceptado más que en un esquema como el que señala Derrida en las lecturas de Jean Rousset sobre Corneille:

Tout se passe comme si, jusqu'en 1643, Corneille n'avait fait qu'entrevoir ou anticiper dans la pénombre le dessin de Polyeucte qui se confondrait avec le dessein cornélien luimême et prendrait ici la dignité d'une entéléchie vers laquelle tout serait 
en marche. Le devenir et le travail cornéliens sont mis en perspective et téléologiquement déchiffrés à partir de ce qui est considéré comme son point d'arrivée, sa structure achevée. Avant Polyeucte, il n'y a que des ébauches dans lesquelles on ne considère que le manque, ce qui au regard de la perfection à venir est encore informe et en défaut; ou encore ce qui annonce seulement la perfection (DERRIDA, 1967, p. 30).

Así, pues, el fantasma se asoma en las grietas del "hombre que anda", como la ausencia de la perfección, o, aún, como la perfección por venir, o igual, en expresión más precisa, un porvenir de la perfección: perfección diferida que no se encuentra en el "hombre que anda" y que cuando se encuentra, o por lo menos cuando se afirma haberla encontrado, ya no es del "hombre que anda", porque éste no es "San Juan Bautista".

Un último salto, hacia el año 1940, nos pone ante la Harmonie, de Aristide Maillol, y a cuatro años de su muerte, en 1944. La visión de la escultura, la mujer sin brazos, nos pone evidentemente ante la Venus de Milo. Unos años antes, ya Magritte, con su Les Menottes de cuivre, de 1931, nos ponía ante una reproducción de esa venus, con el cuerpo pintado en color de "piel", y, dice la anécdota, con tintes en color de sangre en donde se cortan los brazos (SIEBERS, 2008, p. 331). Maillol, aparentemente, no está en sintonía con tal iconoclastía. Delicadamente, se ve a la venus bañándose con serenidad en una pintura suya, Les deux baigneuses (1938), con el detalle sutil de los brazos sumergidos parcialmente en el agua, ocultándolos y produciendo el efecto de "cortarlos", como a los de la venus. Y entorno a la Harmonie, sin embargo, la crónica de su creación nos pone nuevamente ante una situación ambigua. El texto del mismo Musée Maillol la confirma, como ha sido la convención, como una obra "inachevée", e igualmente confirma que el artista usó una modelo para el trabajo, la conocida Dina Vierny (MUSÉE MAILLOL, 2015). El hijo de la modelo, Bertrand Lorquin, hoy un consagrado historiador del arte y conservador del Musée Maillol, ha escrito un estudio profundizado sobre la vida y obra de Maillol. En dicho estudio, con testimonios del artista, se relata la dedicación que puso sobre la Harmonie, empezada en 1940, el trabajo con Vierny y, claramente, que lo "inachevée" de la obra en nada tiene que ver con los brazos, es decir, que de un principio, la escultura sería una venus sin brazos: "“I want this work to be more realist and alive than anything I have done up until now. That is why I am for once working directly from a model, and not just from drawings, as I usually do"” (MAILLOL apud LORQUIN, 1995, p. 148). El crítico e historiador de arte, su biógrafo y amigo, con quien además mantuvo correspondencia, John Rewald, registra su impresión de lo que vio en el proceso del trabajo en la creación de Harmonie: "It became evident to what degree the model was but a guide for the sculptor and with what obstinacy he was pursuing a preconceived idea... one could almost say in spite of the harmonious forms this creature offered him" (REWALD, 1975, p. 27). Si esta 
impresión ha sido una elaboración intelectual para ensalzar póstumamente las visiones del maestro y amigo, no nos parece que se puede juzgar; el hecho es que en las palabras de Maillol recogidas por Lorquin, el sentido de lo afirmado por Rewald encuentra una interesante confirmación:

\footnotetext{
'for Plato, idea and form were identical, and that is how I too understand it. It is a form and a preconceived idea that guides the artist. Followed by gracefulness, power, and all the other qualities which accrue in the course of the work, and as a result the work is not just the realisation of an idea, of an intellectual conception, but is also a work of art' (MAILLOL apud LORQUIN, 1995, p.148).
}

Un triángulo parece formarse, entre modelo, idea y obra. Si, como se dice, la última ha quedado "inachevée" porque Maillol falleció el año 44, y lo inacabado no reside en la incompletud de los brazos, el recorrido de ese camino triangular que sugiere la visión platónica de Maillol se ve acompañado, en el tramo de la idea hacia la obra, del fantasma de la incompletud, el que se realiza en la obra en lugar de la idea. O parte desde la misma concepción de Maillol, se proyecta sobre la modelo y además sobre la idea, y se materializa como la más realizada y bella incompletud. En ambos casos, una renversement du platonsime: el cuerpo desmembrado de la Venus de Milo no es lo que sobró de la más alta realización de lo bello femenino un día alcanzada por la antigüedad, que por accidente se presenta como salió de la tierra en 1810; es la rotura de lo bello aun en su forma ideal, y el eco de esa rotura, o su fantasma, compite de par en par con la idea en la misma mirada que a ésta busca más allá de los bordes rotos:

\footnotetext{
Les copies sont possesseurs en second, prétendants bien fondés, garantis par la ressemblance; les simulacres sont comme les faux prétendants, construits sur une dissimilitude, impliquant une perversion, un détournement essentiels. C'est en ce sens que Platon divise en deux le domaine des images-idoles: d'une part les copiesicônes, d'autre part les simulacres-phantasmes. Nous pouvons alors mieux définir l'ensemble de la motivation platonicienne : il s'agit de sélectionner les prétendants, en distinguant les bonnes et les mauvaises copies, ou plutôt les copies toujours bien fondées, et les simulacres, toujours abîmés dans la dissemblance (DELEUZE, 1969 p. 295-296).
}

\section{Conclusión}

La Harmonie, y el gesto - de un Maillol todavía vivo - que la deja incompleta, pues, estarían bajo el impulso de una época de viraje, así como el de Rodin. Un viraje cuya puesta en curso remota estaría en el momento en que la garantía tranquilizadora de una mirada capaz de trascender de lo material hacia lo ideal introducía lo que negaba en el mismo reino de las formas plenas que pretendía penetrar; o siempre ahí estuvo, como el Sofista se indistingue del 
Filósofo y como la mugre está al lado de lo Bello, en la aporía que acongoja al joven Sócrates ante el viejo Parménides.

El rosto de Ahasuerus, calidoscópico y moviente, nos muestra una narrativa que no existe, cuyo epos no tiene voz que la haga presente sino en los intervalos sin aire del rapsodo, el entretiempo de su respiración, la pausa que retoma el aliento para seguir contando. Pero no hay ahí presencia, si por esa palabra se entiende que es el sujeto de la narrativa, el esto de que se cuenta. Antes, está, pues, el fantasma que se asoma, entre las roturas de la materia y los cortes del tiempo, que de ningún tiempo es, pues no hay uno que sea el suyo. No tiene uno, porque, al fin y al cabo, se asoma como un gesto anónimo, que no se recoge bajo la categoría de un hecho histórico más que como el descubrimiento de un pasado; pero el gesto en si mismo, el que rompió lo que ha sido descubierto, a nadie le pertenece sino a la tierra misma, y sus efectos sobre la mirada, que hemos tratado de señalar aquí y ahí en nuestros saltos, apuntan, tal vez con Nietzsche, hacia aquel "sentido de la tierra", que sólo se encuentra bajando hacia ella:

\begin{abstract}
Nietzsche s'en doutait bien mais Zarathoustra en était sûr : «Me voici entouré de tables brisées et d'autres à demi gravées seulement. Je suis là dans l'attente. Quand viendra mon heure, l'heure de redescendre et de périr...». «Die Stunde meines Niederganges, Unterganges.» Il faudra descendre, travailler, se pencher pour graver et porter la Table nouvelle aux vallées, la lire et la faire lire (DERRIDA, 1967, p. 49).
\end{abstract}

\title{
Referencias
}

ADORNO, Theodor W. Der wünderliche Realist. Über Sigfried Kracauer. In: Noten zur Literatur III. Gesammelte Schriften. Bd.11. Frankfurt am Main: Surhkamp Verlag, 1974. p. 388-408.

AGAMBEN, Giorgio. Estancias. Traducción de Tomás Segovia.Valencia: Pre-textos, 2006.

BAILLIE, Eva Marta. Facing the Fiend: Satan as a Literary Character. Cambridge: Lutterworth, 2014.

BARKAN, Leonard. Unearthing the Past: Archeology and Aesthetics in the Making of Renaissance Culture. New Haven: Yale University Press, 1999.

BUONARROTI, Michelangelo. A Messer Benedetto Varsi. In: MILANESI, Gaetano. Le Lettere di Michelangelo Buonarroti. Pubblicate coi ricordi ed i contratti artistici per cura di Gaetano Milanesi. Firenze: Le Monnier, 1875. p. 522-523.

Non ha l'ottimo artista alcun concetto. In: SYMONDS, John A. The Sonnets of Michaelangelo Buanarotti, now for the first time translated into rhymed English. 2. ed. New York: Smith, Elder, \& Co., C. Scribner's Sons, 1904. p.17. 
CHILVERS, Ian (Ed.). Dicionário Oxford de Arte. Trad. de Marcelo Brandão Cipolla. São Paulo: Martins Fontes, 2007.

CLADEL, Judith (comp.). Auguste Rodin. The man and his art. New York: The Century Co., 1917.

CONCHA, Jaime. El Aleph: Borges y la Historia. Revista Iberoamericana, Pittsburg, v.XLIX, n. 123-124, p. 471-485, abr-sept. 1983.

DELEUZE, Gilles. Logique du sens. Paris: Minuit, 1969.

DERRIDA, Jacques. L'ecriture et la différence. Paris: Seuil, 1967.

GOMBRICH, Ernst Hans. The Story of Art. London: Phaidon, 1989.

JANSON, Anthony. History of Art. The Western Tradition. London: Pearson, 2004.

KRACAUER, Sigfried. History: The Last Things Before the Last. New York: Oxford University Press, 1969.

KUBLER, Geroge. The Shape of Time: Remarks on the History of Things. New Haven: Yale University Press, 2008.

LORQUIN, Bertrand. Aristide Maillol. New York: Skira - Thames \& Woodson, 1995.

MUSÉE MAILLOL. Fondation Dina Verny. Disponible en: $<$ http://www.museemaillol.com/le-musee-maillol/le-musee-de-paris/>. Accedido en: 2 de feb. 2015.

POTTS, Alex. Flesh and Ideal: Winckelmann and the origins of Art History. New Haven: Yale University Press, 1994.

REWALD, John. Maillol remembered. In: GUGGENHEIM MUSEUM. Aristide Maillol: 1861-1944: exhibition. New York: The Solomon R. Guggenheim Foundation, 1975. p.7-31.

RODOWICK, David N. The Last Things before the Last: Kracauer and History. New German Critique, Duke University, n.41, p.109-139, Spring-Summer 1987.

SCHARFSTEIN, Ben-Ami. Art without borders. A philosophical exploration of Art and Humanity. Chicago: The University of Chicago Press, 2009.

SIEBERS, Tobin. Disability aesthetics and the body beautiful: Signposts in the history of art. ALTER, European Journal of Disability Research. v. 2. 2008. p. 329-336.

STOKES, Adrian. Michelangelo. New York: Routledge, 2002.

SUMMERS, David. Contrapposto: Style and Meaning in Renaissance Art. The Art Bulletin, College Art Association, v. 59, n. 3, p. 336-361, September 1977.

TAYLOR, John Edward. Michael Angelo, considered as a Philosophic Poet; with translations, etc. London: Saunders and Otley, 1840. 
VASARI, Giorgio. Le vite de' più eccellenti pittori, scultori e architettori. A cura di Luciano Bellosi e Aldo Rossi. Einaudi: Torino, 1986.

WEINBERG, Louis. The art of Rodin. New York: Boni and Liveright Publishers, 1918.

WINCKELMANN, Johan Joachim. Geschichte der Kunst des Alterthums. In: LESSING, Julius. Johann Joachim Winckelmann's Geschichte Der Kunst Des Alterthums: Nebst Einer Auswahl Seiner Kleineren Schriften; Mit Einer Biographie Winckelmanns Und Einer Einleitung Versehen. Berlin: L. Heimann Verlag, 1870. p.15-354.

WINCKELMANN, Johann Joachin. Entwürfe zur Beschreibng des Torso im Belvedere. In: REHM, Walter (Ed.). Johann J. Winckelmann. Kleine Schriften. Vorreden. Entwürfe. Berlin: De Gruyter, 2002. p. 280-5.

[Recebido em abril de 2015 e aceito para publicação em junho de 2015]

\section{The phantasm of the cracks: an an-historic speculation}

Abstract: From Siegfried Kracauer's and George Kubler's concept of "shape of time", we essay a reading of the reception of the mutilated body image in art, analysing how discourses of historians, artists and critics articulate around that image in certain moments of history. We suggest, with Agamben, Derrida, and Deleuze, that a phantasm might be haunting the body image in art, whose spectrality manifests itself underneath the very discourses trying to repress it. We visit the Renaissance, the XVIIIth century, and the turning from the XIXth to the XXth. We conclude that a change of attitude in the XXth century is the reflex of the overturning of platonism which was being announced since the XIXth century, but whose symptoms were felt since before, manifested in a contradictory manner in discourses which praise the beauty of the fragment in the same time they use it to reach an image of beauty beyond the material realm.

Keywords: Art. Body. Image. Phantasm. History.

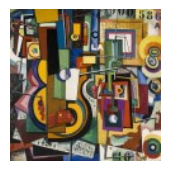

